\title{
Location of collinear equilibrium points in the generalised photogravitational elliptic restricted three body problem
}

\author{
Sanjay Kumar $^{1} *$, B.Ishwar ${ }^{2}$ \\ ${ }^{1 *}$ Department of Mathematics, R.D.S.College, Muzaffarpur, Bihar, INDIA \\ ${ }^{2}$ P.I., D.S.T., Project, University Department of Mathematics, B.R.A.Bihar University, Muzaffarpur, Bihar, INDIA \\ "Corresponding Author: e-mail: dr.sanjaykumar06@gmail.com,Tel +91-9234370450
}

\begin{abstract}
We have discussed the location of collinear equilibrium points in the generalised photogravitational restricted three body problem. The problem is generalised in the sense that both primaries are oblate spheroid. They are source of radiation as well. We have found the solution for the location of collinear point $\mathrm{L}_{1}$. We found that location of collinear point $\mathrm{L}_{1}$ is affected by eccentricity, oblateness and radiation factor terms. The same method may be applied for location of collinear points $\mathrm{L}_{2}$ and $\mathrm{L}_{3}$.
\end{abstract}

Keywords: Collinear points, generalised photogravitational, ERTBP.

\section{Introduction}

Radzievskii (1950) formulated the photogravitational restricted three body problem. This arises from the classical problem when one of the masses is an intense emitter of radiation. Arnold (1961) studied the stability of positions of equilibrium of a Hamiltanian system in the general elliptic case. Chaudhary (1966) studied the periodic orbits of the third kind and stability of the generating solution in the elliptical restricted three body problem. Bhatanagar (1969) examined periodic orbits of collision in the elliptic restricted three body problem. Sharma and Subbarao (1975) studied the restricted three body problem when the primaries are oblate spheroids. Sharma (1982) investigated the linear stability of triangular libration points when the more massive primary is a source of radiation and oblate spheroid as well. He also examined the linear stability of libration points of the photogravitational restricted three body problem when the smaller primary is an oblate spheroid. Beauge (1996) gave a note on a global expansion of the disturbing function in the planar elliptic restricted three body problem. Khasan (1996) studied librational solutions to the photogravitational restricted three body problem by considering both primaries as radiating. He also examined the stability of collinear and triangular points. Khasan (1996a) also studied three dimensional periodic solutions to the photogravitational Hill problem. He investigated restricted photogravitational elliptic three body problem. Sahoo and Ishwar (2000) studied the stability of collinear equilibrium points in the generalised photogravitational elliptic three body problem. Kumar and Ishwar (2004) studied the equations of motion in the generalised photogravitational elliptic restricted three body problem. Kumar and Ishwar (2009) investigated the solutions of generalised photogravitational elliptic restricted three body problem.

Hence, we thought to establish location of collinear equilibrium points in the generalised photogravitational elliptic restricted three body problem. In restricted three body problem, when the primaries move on ellipse is called ERTB (elliptic restricted three body problem). The problem is generalised in the sense that both primaries are considered as oblate spheroid. They are source of radiation as well.

\section{Location of Collinear Points}

The equilibrium points are the solutions of the equations:

This paper is presented in $5^{\text {th }}$ National Conference on Applicable Mathematics in Wave Mechanics and Vibrations (WMVC2010) held at Kakatiya University, Warangal, India,13-15 March,2010. 


$$
\frac{\partial f}{\partial \xi}=\frac{\partial f}{\partial \eta}=\frac{\partial f}{\partial \zeta}=0
$$

where

$$
f=\frac{1}{\left(1-e^{2}\right)^{1 / 2}}\left[\frac{\xi^{2}+\eta^{2}}{2}+\frac{1}{n^{2}}\left\{\frac{(1-\mu) q_{1}}{r_{1}}+\frac{\mu q_{2}}{r_{2}}+\frac{(1-\mu) q_{1} A_{1}}{2 r_{1}^{3}}+\frac{\mu q_{2} A_{2}}{2 r_{2}^{3}}\right\}\right]
$$

is the force function in the equation of motion of our problem.

and

$$
r_{1}^{2}=\left(\xi-\xi_{1}\right)^{2}+\eta^{2}+\zeta^{2}
$$

$$
\begin{aligned}
& r_{2}^{2}=\left(\xi-\xi_{2}\right)^{2}+\eta^{2}+\zeta^{2} \\
& \mu=\text { mass parameter } \\
& q_{1}=\text { radiation parameter of } \mathrm{m}_{1} \\
& q_{2}=\text { radiation parameter of } \mathrm{m}_{2} \\
& A_{1}=\text { oblateness parameter of } m_{1} \\
& A_{2}=\text { oblateness parameter of } m_{2} \\
& n=\text { average angular velocity } \\
& e=\text { eccentricity of the orbit. }
\end{aligned}
$$

The equation $\frac{\partial f}{\partial \xi}=\frac{\partial f}{\partial \eta}=\frac{\partial f}{\partial \zeta}=0$ gives

$$
\begin{aligned}
& \xi-\frac{1}{n^{2}}\left\{\frac{(1-\mu) q_{1}\left(\xi-\xi_{1}\right)}{r_{1}^{3}}+\frac{\mu q_{2}\left(\xi-\xi_{2}\right)}{r_{2}^{3}}+\frac{3(1-\mu) q_{1} A_{1}\left(\xi-\xi_{1}\right)}{2 r_{1}^{5}}+\frac{3 \mu q_{2} A_{2}\left(\xi-\xi_{2}\right)}{2 r_{2}^{5}}\right\}=0 \\
& \eta\left\{1-\frac{1}{n^{2}}\left(\frac{(1-\mu) q_{1}}{r_{1}^{3}}+\frac{\mu q_{2}}{r_{2}^{3}}+\frac{3(1-\mu) q_{1} A_{1}}{2 r_{1}^{5}}+\frac{3 \mu q_{2} A_{2}}{2 r_{2}^{5}}\right)\right\}=0 \\
& -\frac{\zeta}{n^{2}}\left(\frac{(1-\mu) q_{1}}{r_{1}^{3}}+\frac{\mu q_{2}}{r_{2}^{3}}+\frac{3(1-\mu) q_{1} A_{1}}{2 r_{2}^{3}}+\frac{3 \mu q_{2} A_{2}}{2 r_{2}^{2}}\right)=0
\end{aligned}
$$

For collinear equilibrium points lying on the line joining the primaries i.e. on $\xi$-axis, we have from equation (2)

or,

$$
\xi-\frac{1}{n^{2}}\left(\frac{(1-\mu) q_{1}\left(\xi-\xi_{1}\right)}{\left[\left(\xi-\xi_{1}\right)^{2}\right]^{3 / 2}}+\frac{\mu q_{2}\left(\xi-\xi_{2}\right)}{\left[\left(\xi-\xi_{2}\right)^{2}\right]^{3 / 2}}+\frac{3(1-\mu) q_{1} A_{1}\left(\xi-\xi_{1}\right)}{2\left[\left(\xi-\xi_{1}\right)^{2}\right]^{5 / 2}}+\frac{3 \mu q_{2} A_{2}\left(\xi-\xi_{2}\right)}{2\left[\left(\xi-\xi_{2}\right)^{2}\right]^{5 / 2}}\right)=0
$$

$$
\begin{aligned}
& 2\left(\xi-\xi_{1}\right)^{2}\left(\xi-\xi_{2}\right)^{2}\left\{n^{2} \xi\left(\xi-\xi_{1}\right)^{2}\left(\xi-\xi_{2}\right)^{2}-(1-\mu) q_{1}\left(\xi-\xi_{2}\right)^{2}-\mu q_{2}\left(\xi-\xi_{1}\right)^{2}\right\} \\
& -3(1-\mu) q_{1} A_{1}\left(\xi-\xi_{2}\right)^{4}-3 \mu q_{2} A_{2}\left(\xi-\xi_{1}\right)^{4}=0
\end{aligned}
$$


This is ninth degree equation, so we shall get nine roots of $\xi$. Three equilibrium points lie on the $\mathrm{X}$-axis. One root of $\xi$ is greater than $\xi_{2}$, other root lies between $\xi_{1}$ and $\xi_{2}$ and the third root is less than $\xi_{1}$ :

\section{Case I}

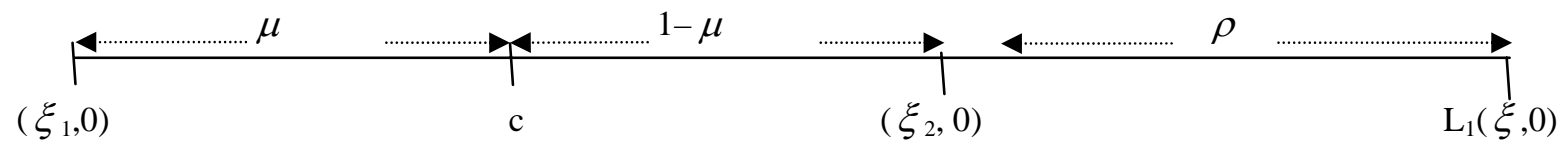

In this case $\xi>\xi_{2}$ and we consider

Therefore

$$
\xi-\xi_{2}=\rho
$$

Hence

$$
\xi-\xi_{1}=1+\rho
$$

$$
\xi_{2}-\xi_{1}=1
$$

Since $\mathrm{c}$ is the centre of mass

$$
(1-\mu) \xi_{1}+\mu \xi_{2}=0
$$

which gives

and

$$
\xi_{1}=-\mu
$$

$$
\xi=1+\rho+\xi_{1}=1+\rho-\mu
$$

Substituting these values in the equation (5), we get

$$
\begin{aligned}
& 2 n^{2} \rho^{9}+2 n^{2}(5-\mu) \rho^{8}+2 n^{2}(10-4 \mu) \rho^{7}+\left[2 n^{2}(10-6 \mu)-2 q_{1}(1-\mu)-2 \mu q_{2}\right] \rho^{6}+ \\
& {\left[2 n^{2}(5-4 \mu)-4 q_{1}(1-\mu)-8 \mu q_{2}\right] \rho^{5}+\left[2 n^{2}(1-\mu)-2 q_{1}(1-\mu)-12 \mu q_{2}-3(1-\mu) q_{1} A_{1}-3 \mu q_{2} A_{2}\right] \rho^{4}} \\
& -\left[8 \mu q_{2}+12 \mu q_{2} A_{2}\right] \rho^{3}-\left[2 \mu q_{2}+18 \mu q_{2} A_{2}\right] \rho^{2}-12 \mu q_{2} A_{2} \rho-3 \mu q_{2} A_{2}=0
\end{aligned}
$$

For verification we put $n=1, q_{1}=1, q_{2}=1, A_{1}=0, A_{2}=0$ we get the same result as in classical case. Let $\gamma_{1}$ be the solution of the classical case i.e. when $e=0, A_{1}=A_{2}=0, q_{1}=q_{2}=1$, and due to the presence of these terms the location will be slightly changed

Let the new value of $\rho$ be defined by $\rho=\gamma_{1}+\delta$, where $\delta$ is very-very small and let $q_{1}=1-\beta_{1}, q_{2}=1-\beta_{2}$ where $\beta_{1}$, $\beta_{2}$ $<<1$. Substituting the value of $\rho$ in the equation (6), we get

$$
\begin{aligned}
\rho=\gamma_{1}+\delta= & \gamma_{1}+\left[U_{1} X_{1}+\left(U_{1} Y_{1}+V_{1} X_{1}\right) e^{2}+\left(U_{1} Y_{1}+V_{1} X_{1}\right) A_{1}+\left(U_{1} Y_{1}+V_{1} X_{1}\right) A_{2}\right]+ \\
& \left(X_{1}+Y_{2} e^{2}+y_{1} A_{1}+Y_{1} A_{2}\right)\left[-2(1-\mu)\left\{\left(\gamma_{1}^{6}+2 \gamma_{1}^{5}+\gamma_{1}^{4}\right)+\left(6 \gamma_{1}^{5}+10 \gamma_{1}^{4}+4 \gamma_{1}^{3}\right) U_{1} X_{1}\right\}\right] \beta_{1}+ \\
& \left.\left.\left(6 \gamma_{1}^{5}+20 \gamma_{1}^{4}+24 \gamma_{1}^{3}+12 \gamma_{1}^{2}+2 \gamma_{1}\right) U_{1} X_{1}\right\}\right] \beta_{2}+ \\
& \left(X_{1}+Y_{1} e^{2}+Y_{1} A_{1}+Y_{1} A_{2}\right)\left[3(1-\mu)\left\{\left(\gamma_{1}^{4}+4 \gamma_{1}^{3}\right) U_{1} X_{1}\right\}\right] A_{1}+ \\
& \left(X_{1}+Y_{1} e^{2}+Y_{1} A_{1}+Y_{1} A_{2}\right)\left[3 \mu\left\{\gamma_{1}^{4}+4 \gamma_{1}^{3}+6 \gamma_{1}^{2}+4 \gamma_{1}+1\right)\right. \\
& \left.\left.+\left(4 \gamma_{1}^{3}+12 \gamma_{1}^{2}+12 \gamma_{1}+4\right) U_{1} X_{1}\right\}\right] A_{2}
\end{aligned}
$$


where $\gamma_{1}$ is the value of $\rho$, the distance between $\mathrm{L}_{1}$ and smaller primary in the classical case. We have found the value of $U_{1}, V_{1}, X_{1}, Y_{1}$ as in Sahoo and Ishwar (2000),

$$
\begin{aligned}
U_{1}= & \frac{1}{a}\left[-2 \gamma_{1}^{9}-2(5-\mu) \gamma_{1}^{8}-2(10-4 \mu) \gamma_{1}^{7}-2(10-6 \mu) \gamma_{1}^{6}-2(5-4 \mu) \gamma_{1}^{5}-2(1-\mu) \gamma_{1}^{4}\right] \\
& +2 \gamma_{1}^{6}+4(1+\mu) \gamma_{1}^{5}+2(1+5 \mu) \gamma_{1}^{4}+8 \mu \gamma_{1}^{3}+2 \mu \gamma_{1}^{2} \\
V_{1}= & \frac{-3}{a}\left[\gamma_{1}^{9}+(5-\mu) \gamma_{1}^{8}+(10-4 \mu) \gamma_{1}^{7}+(10-6 \mu) \gamma_{1}^{6}+(5-\mu) \gamma_{1}^{5}+(1-\mu) \gamma_{1}^{4}\right] \\
X_{1}= & {\left[\frac{1}{a}\left\{18 \gamma_{1}^{8}+16(5-\mu) \gamma_{1}^{7}+28(5-2 \mu) \gamma_{1}^{6}+24(5-3 \mu) \gamma_{1}^{5}+10(5-4 \mu) \gamma_{1}^{4}+8(1-\mu) \gamma_{1}^{3}\right\}\right.} \\
& \left.-\left\{12 \gamma_{1}^{5}+20(1+\mu) \gamma_{1}^{4}+8(1+5 \mu) \gamma_{1}^{3}+24 \mu \gamma_{1}^{2}+4 \mu \gamma_{1}\right\}\right]^{-1} \\
& {\left[\begin{array}{rl}
- & \left.\frac{1}{a}\left\{27 \gamma_{1}^{8}+24(5-\mu) \gamma_{1}^{7}+42(5-2 \mu) \gamma_{1}^{6}+36(5-3 \mu) \gamma_{1}^{5}+15(5-4 \mu) \gamma_{1}^{4}+12(1-\mu) \gamma_{1}^{3}\right\}\right] \\
Y_{1}= & {\left[\frac{1}{a}\left\{18 \gamma_{1}^{8}+16(5-\mu) \gamma_{1}^{7}+28(5-2 \mu) \gamma_{1}^{6}+24(5-3 \mu) \gamma_{1}^{5}+10(5-4 \mu) \gamma_{1}^{4}+8(1-\mu) \gamma_{1}^{3}\right\}\right.} \\
& \left.\left[12 \gamma_{1}^{5}+20(1+\mu) \gamma_{1}^{4}+8(1+5 \mu) \gamma_{1}^{3}+24 \mu \gamma_{1}^{2}+4 \mu \gamma_{1}\right\}\right]
\end{array}\right.}
\end{aligned}
$$

$a$ =semi-major axis of ellipse.

Hence, we located collinear point $L_{1}$.

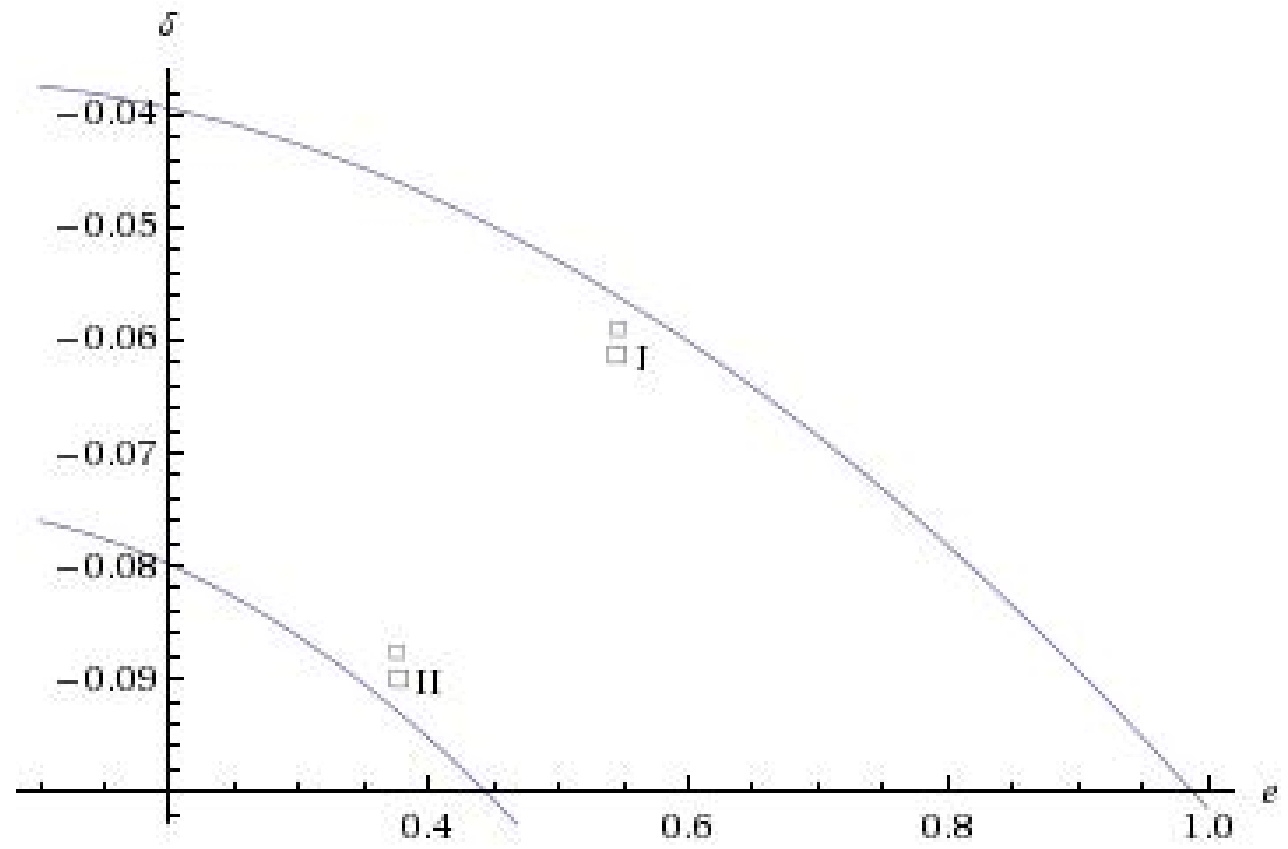

Fig.-1: Effect of $A_{1}, A_{2}, q_{1}$ and $q_{2}$ on the position of $L_{1}$ 


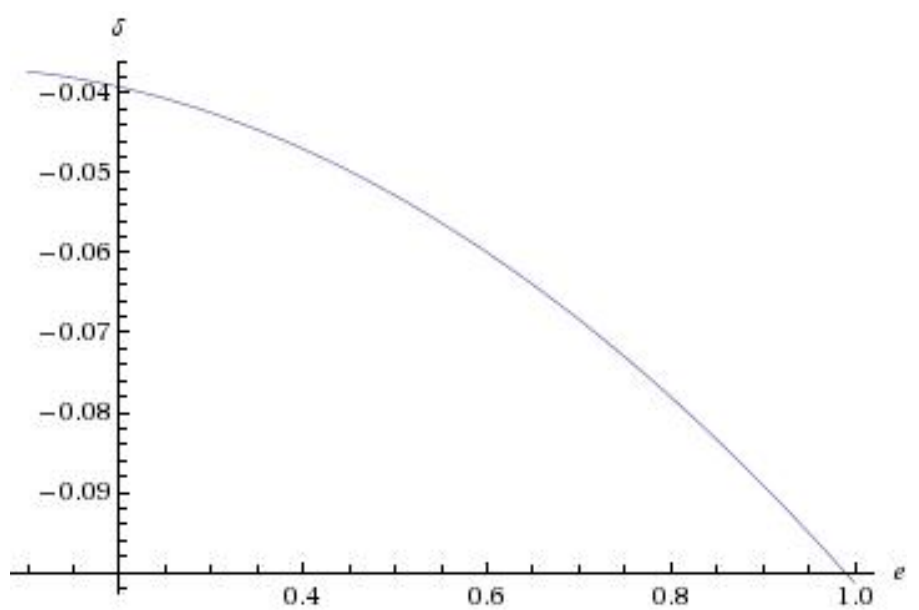

(a)

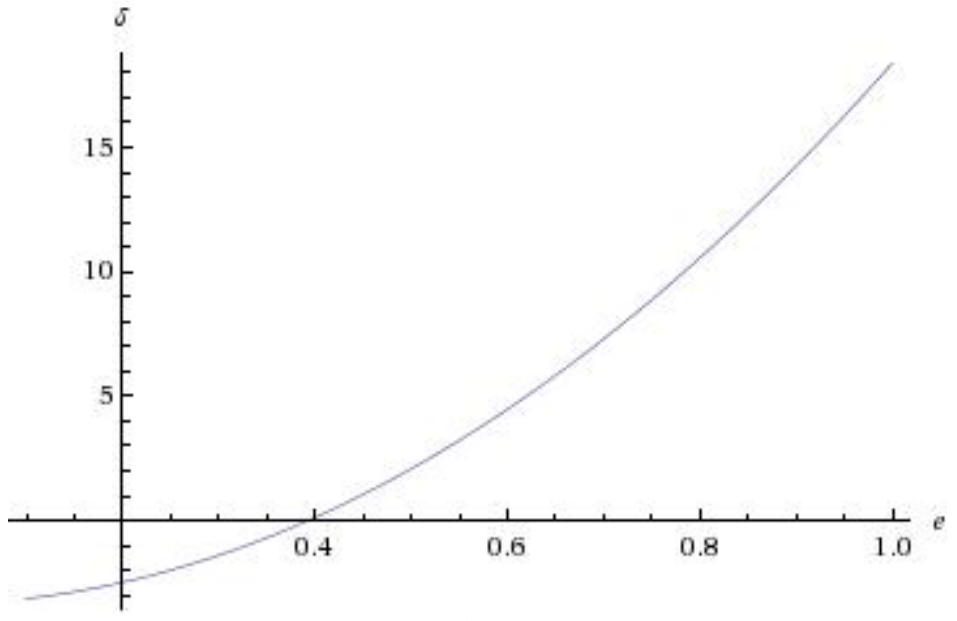

(b)

Fig.-2:Effect of mass parameter $\mu$ on the position of $L_{1}$

For numerical calculation we have used $\mathrm{a}=.0001, \beta_{1}=\beta_{2}=0.0001$, and plotted the above graphs for deviation $\delta$ Vs eccentricity. In figure 1 we consider $A_{1}=0.0025$ and $\mu=0.00025$, where curve I represents the effect of oblateness coefficients $A_{2}=0.0025$ and curve II for $A_{2}=0.0050$. The effect of mass parameter $\mu$ is depicted in figure 2 with (a): $\mu=0.00025$ and (b): $\mu=0.0025$. One can see that the deviation $\delta$ in (a) is decreasing while in (b) this $\delta$ is increasing with eccentricity e. We have also computed the effect of $\beta_{1}, \beta_{2}$ on the position of $L_{1}$ but this effect is very insignificant, and the graphs are similar to the above figures even if the values of $A_{1}, A_{2}$ are interchanged.

\section{Conclusion}

Thus, we conclude that collinear point $\mathrm{L}_{1}$ is affected by oblateness, eccentricity and radiation factor terms. Also when these terms are neglected, we get the same terms as in the classical elliptical restricted three body problem. The same method may be applied for location of collinear points $L_{2}$ and $L_{3}$. Numerically we have obtained that the position of $L_{1}$ is deviated from classical case for various values of parameters.

\section{References}

Arnold V.I., 1961. On the stability of positions of equilibrium of a Hamiltonian system of ordinary differential equations in the general elliptic case. Soviet Math. Vol. 2, p. 247. 
Bhatnagar, K.B., 1969. Periodic orbits of collision in the plane elliptic restricted problem of three bodies. Proc. Natn. Inst. Sci. India 35A, pp. 829-44

Beauge, C., 1996. Cales Mech. and Dyn. Astron. Vol 64, No. 4.

Chaudhary, R.K., 1966. Existence of periodic orbits of the third kind in the elliptical restricted three body problem and stability of the generating solution. Bull. Ins. Theoret. Astronomy Vol. 10, pp. 523-536.

Danby, J.M.A., 1964. Stability of triangular points in the Elliptic Restricted problem of three bodies. Astronomical Journal Vol. 69, pp. 165-172.

Khasan, S.N., 1996. Librational solutions to the photogravitational restricted three body problem. Cosmic Research, Vol. 34, No.2, pp. 146-151.

Khasan, S.N., 1996a. Cosmic Research, Vol. 34, No. 5, pp. 504-507.

Kumar, Sanjay and Ishwar, B., 2004. Equations of motion in the generalized photogravitational elliptic restricted three body problem. Review Bull. Cal. Math. Soc., Vol. 12, No. (1 and 2), pp. 115-118.

Kumar, Sanjay and Ishwar, B., 2009. Solutions of generalized photogravitational elliptic restricted three body problem. AIP Conference Proceedings, Vol. 1146, pp. 456-461.

Radzievskii, V.V., 1950. The restricted problem of three bodies taking account of light pressure. Akad. Nauk. USSR, Astron, Journal Vol. 27, p. 250.

Sahoo, S.K. and Ishwar,B, 2000. Stability of collinear equilibrium points in the generalized photogravitational elliptic three body problem. Bull. Astr. Soc. India, Vol. 28, p. 579.

Sharma, R.K., W.Frucke and G.Teleki (eds), 1982. Sun and Planetary system, Vol. 435, D. Reidel Publishing company,

\section{Biographical notes}

Dr. Sanjay Kumar is a Senior Lecturer in the P.G. Department of Mathematics, R.D.S. College, Muzaffarpur, B.R.A.Bihar University, Muzaffarpur, Bihar, India. He has more than 13 years of experience in teaching. He has published six papers in referred national and international journals. He has also presented several papers in national and international conferences.

B.Ishwar (D.Sc.) University Professor is a Principal Investigator, D.S.T. Project, University Department of Mathematics, B.R.A.Bihar University, Muzaffarpur, Bihar, India.

Received September 2010

Accepted June 2011

Final acceptance in revised form June 2011 